\title{
Grappling with family practice complexities
}

I nterdisciplinary health teams, chronic disease management, responsible prescribing of pain-management drugs and evidence-based clinical practice are top of mind for many family physicians heading to the profession's annual general meeting.

"The issues that we'll talk about or bring up can be divided into three different sections: clinical, education and research," says Dr. Pierre-Paul Tellier, chair of the advisory committee for the Family Medicine Forum, held in Toronto, Ontario, from Nov. 15-17.

Several of the concerns are reflections of the growing complexity of family medicine and the difficulties in delivering health care in a fashion appropriate to the nation's various demographic needs, according to provincial representatives to the conference, which is cohosted by the College of Family Physicians of Canada and the Ontario College of Family Physicians.

Yet, while meeting basic health needs of a small, widely dispersed population, such as that of Nunavut, can present unique challenges, oft-times the obstacles are universal, as with providing care effectively through interdisciplinary health teams, to the aging population and to the increasing number of people with chronic diseases.

"Chronic disease management is complex. In order for a family doctor to do it, you need help," says Dr. Charlene Fitzgerald, president of the Newfoundland and Labrador College of Family Physicians. "You need help from other medical professionals to manage and organize the care. The help we need is team-based care, interdisciplinary teams organized around a hub - the patient."

That opinion is shared by many Canadian physicians, even those who work more than 5000 kilometres from Fitzgerald's practice in Goose Bay, Newfoundland and Labrador. But there's no consensus on the best means of turning the notion of team-based care into reality.

"The concept may be broadly

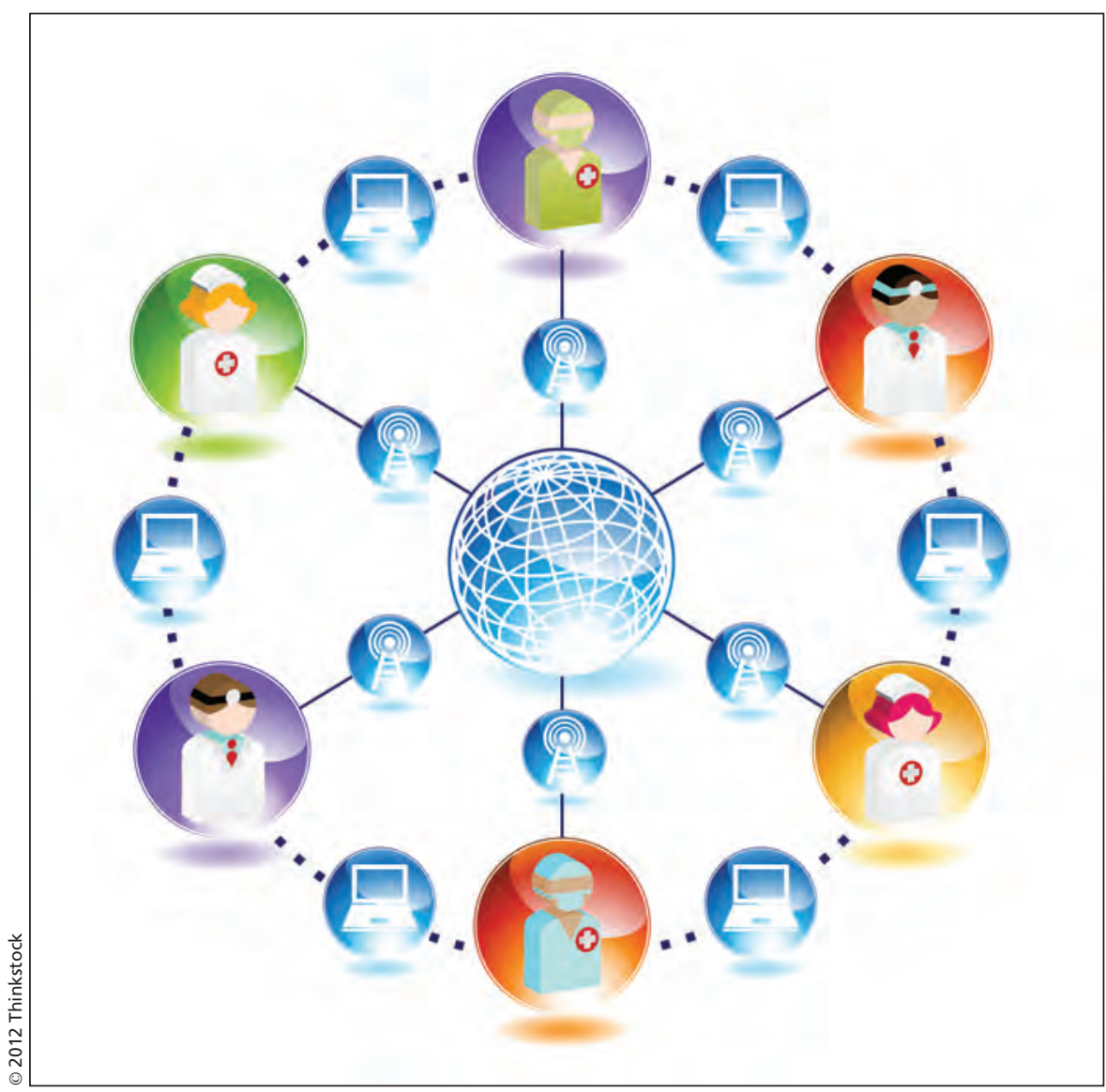

Converting the notion of team-based care into a reality is often problematic for physicians.

accepted, but it's one thing to have an idea and another thing to make it work in practice," says Dr. Jim Thorsteinson, executive director of the British Columbia College of Family Physicians.

Ontario and Alberta are often cited as being in the vanguard of team-based care but even they have struggled to implement the notion. "How do we implement some of these things we would like to do?" notes Dr. Cathy Scrimshaw, president of the Alberta College of Family Physicians. "Some practices are well-suited for the change, some already have interdisciplinary teams, but some aren't involved in that at all. One of the things I hear the most is: How do I fit, physically, a team into my practice?"

Another topic expected to attract considerable interest at the forum is respon- sible prescribing practices, particularly as the misuse, abuse and diversion of prescription drugs are problems that show no signs of abating and the federal and provincial governments are in a bit of a tussle over whether generic oxycodone should be allowed onto the market once the patent on the brand-name version expires Nov. 25 (www.cmaj.ca /lookup/doi/10.1503/cmaj.109-4326).

"One thing I've seen a lot of focus on is safe and effective use of pain-management medications," says Thorsteinson. "It's a challenging area."

The issue is complex because so many factors come into play, including addiction, mental health and biological variance in response to medication. Several presentations on the issue will be made at the forum, including one sketching a pain and addictions primer 
and another on how to taper opioids and address problem drug use in patients with chronic pain.

Getting more clinical value out of medical research is another concern for many family physicians, as the translation from academic research to on-theground know-how is often lacking, says Scrimshaw. "How do we distill it down to get to the quality of the evidence in a form that is practical for us to use in our practices?"

Tellier expects there'll also be considerable debate around new clinical guidelines, sports-related injuries and various community health needs. "There has been more emphasis on encouraging people to be involved in sporting activities, and when dealing with a population that is more active, whether they are young or old, they will have aches and pains that need to be managed."

Among issues that Tellier believes need to be addressed in more detail in the future are physician wellness and the effective use of technology to improve health outcomes. "It is also my hope that we bring some more acade- mic rigour to the whole meeting. We are still a relatively young conference, only about 10 years old, and we were learning how to work it, how to deliver a product. Now we have to learn how to improve the quality of that product."

Roughly 4500 doctors, educators, researchers, medical students, residents and other health professionals attended the 2011 forum, making it the fastest growing, and largest, medical gathering in Canada. — Roger Collier, CMAJ

CMAJ 2012. DOI:10.1503/cmaj.109-4348 